\title{
Crystal Structure of Diels-Alder Cycloadduct Formed from 1-(1,2,3-1H- Benzotriazol-1-yl)-2-(4-methylphenyl)-2H-isoindole and Dimethyl Acetylenedicarboxylate
}

\author{
Ichiro Takahashi, ${ }^{*}$ Mikio Tsuzuki,* Hidehiko Kitajima,* Minoru Hatanaka,* Shiro Maeda,* \\ Akihito Yamano, $* *$ Tomihisa OHTA, ${ }^{* * *}$ and Shinzo Hosol ${ }^{* * * *}$ \\ *Department of Applied Chemistry and Biotechnology, Faculty of Engineering, Fukui University, \\ 3-9-1 Bunkyo, Fukui 910-8507, Japan \\ **X-ray Research Laboratory, Rigaku Corporation, 3-9-12 Matsubara-cho, Akishima, \\ Tokyo 196-8666, Japan \\ ***Faculty of Pharmaceutical Sciences, Kanazawa University, 13-1 Takara-machi, Kanazawa 920-0934, Japan
}

\begin{abstract}
The structure of the Diels-Alder cycloadduct formed from 2-(1,2,3-1H-benzotriazol-1-yl)-2-( $p$-tolyl)-2H-isoindole and dimethyl acetylenedicarboxylate was proved as 11-aza-1-(1,2,3-1H-benzotriazol-1-yl)-11-(4-methylphenyl)-tricyclo[5.2.1.0 $\left.0^{2,7}\right]$ undeca-2,4,6,9-tetraene-9,10-dioic acid dimethyl ester. The benzotriazole moiety was located as its 1-yl form, analogous to previous reports. The benzotriazole and the benzene (of tricyclo framework) planes were twisted with an angle of $115.83^{\circ}$. Intramolecular close contacts between benzotriazole and ester are characteristic $[\mathrm{N}(3) \cdots \mathrm{C}(26)$, 2.754(3) $\mathrm{A} ; \mathrm{N}(3) \cdots \mathrm{H}(22), 3.26(4) \AA]$. The shortest contact of $\mathrm{N}(3) \cdots \mathrm{H}(22)$ accounting for the rotation of the methyl group is estimated to be $3.10 \AA$, which might be reasonable as $\mathrm{C}-\mathrm{H} \cdot \cdots \mathrm{N}$-type hydrogen bonding.
\end{abstract}

(Received September 24, 2002; Accepted February 3, 2003)

We previously reported on the dual characters of benzotriazolesubstituted isoindole derivatives, which turned out to be a rather stable crystalline material in spite of its high reactivity. ${ }^{1}$ During our studies on the utility of these "stable" isoindoles as a diene for Diels-Alder cycloaddition, it proved to be difficult to determine the structure of the cycloadduct by the NMR method. This is presumably because (i) the ${ }^{1} \mathrm{H}$ signal pattern of cycloadduct in an aromatic region is very different from that of the previously reported benzotriazole-containing compounds, and thus analogical assignments are impossible; $; 2,3$ (ii) it is hard to apply 2D techniques in NMR for correlations across four consecutive tertiary carbons units and $\mathrm{N}-\mathrm{N}=\mathrm{N}$ linkages; (iii) one of the ester methyl groups appears as a broad signal in the ${ }^{1} \mathrm{H}$ NMR spectrum, suggesting the existence of a rotational barrier at room temperature, the cause of which has not been specified. In this paper we thus wish to report on the crystal structure of the title compound (1).

A cycloaddition reaction of 1-(1,2,3-1H-benzotriazol-1-yl)-2(4-methylphenyl)- $2 \mathrm{H}$-isoindole with dimethyl acetylenedicarboxylate was performed in chloroform at room temperature to give crude $\mathbf{1}$ which was purified by recrystallization from boiling methanol to give pure $\mathbf{1}$ as paleyellow prisms (m.p. $150-153^{\circ} \mathrm{C}$ ). The crystal and experimental data are listed in Table 1. The non-hydrogen atoms were refined anisotropically. All of the hydrogen atoms were located from a difference Fourier map and included in the final cycle of the full-matrix least-squares refinements. The positional parameters for non-hydrogen atoms are listed in Table 2.

$\dagger$ To whom correspondence should be addressed.

E-mail: hosoi@kenroku.kanazawa-u.ac.jp
Table 1 Crystal and experimental data

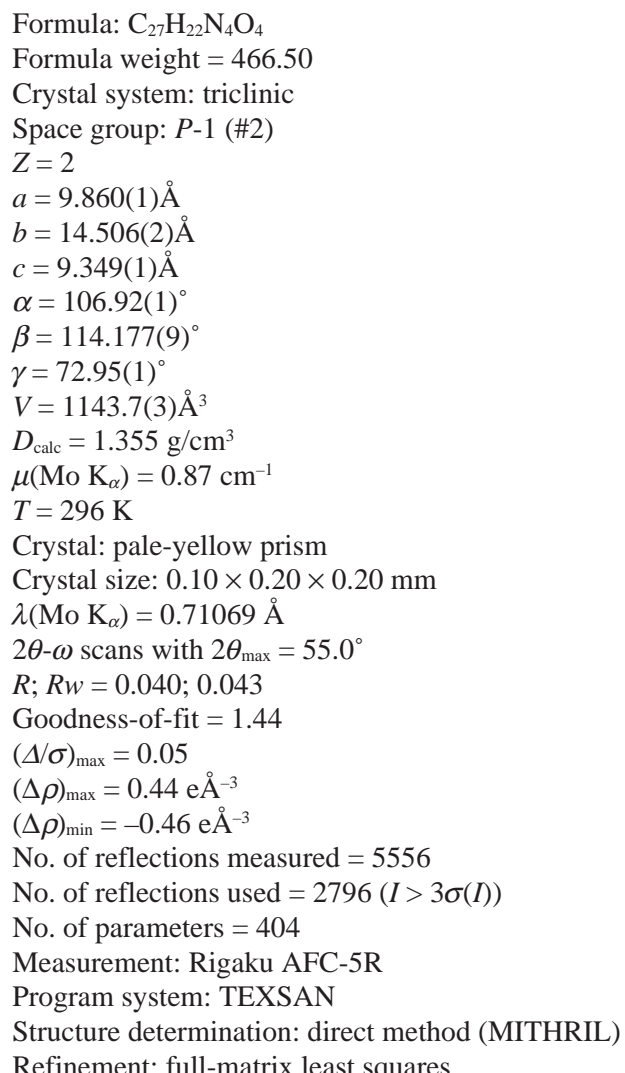

Refinement: full-matrix least squares 
Table 2 Fractional coordinates and thermal parameters for non-hydrogen atoms

\begin{tabular}{|c|c|c|c|c|}
\hline Atom & $x$ & $y$ & $z$ & $B_{\mathrm{eq}} / \AA^{2}$ \\
\hline$O(1)$ & $1.6307(2)$ & $0.1187(1)$ & $0.6415(2)$ & $5.8(1)$ \\
\hline$O(2)$ & $1.6003(2)$ & $0.2685(1)$ & $0.7923(2)$ & $3.93(7)$ \\
\hline$O(3)$ & $1.2732(2)$ & $0.4586(1)$ & $0.7185(2)$ & $4.89(9)$ \\
\hline$O(4)$ & $1.3471(2)$ & $0.3729(1)$ & $0.9119(2)$ & $3.78(7)$ \\
\hline$N(1)$ & $1.1521(2)$ & $0.1585(1)$ & $0.4852(2)$ & $3.00(8)$ \\
\hline$N(2)$ & $0.9959(2)$ & $0.3202(1)$ & $0.5636(2)$ & $2.97(8)$ \\
\hline$N(3)$ & $1.0143(2)$ & $0.3682(1)$ & $0.7179(2)$ & $3.51(9)$ \\
\hline $\mathrm{N}(4)$ & $0.8813(2)$ & $0.4010(2)$ & $0.7307(2)$ & $3.8(1)$ \\
\hline$C(1)$ & $1.1266(2)$ & $0.2696(2)$ & $0.5193(3)$ & $2.78(9)$ \\
\hline$C(2)$ & $1.1251(2)$ & $0.2835(2)$ & $0.3633(3)$ & $3.1(1)$ \\
\hline$C(3)$ & $1.0517(3)$ & $0.3559(2)$ & $0.2748(3)$ & $3.7(1)$ \\
\hline$C(4)$ & $1.0836(3)$ & $0.3446(2)$ & $0.1375(3)$ & $4.4(1)$ \\
\hline$C(5)$ & $1.1833(3)$ & $0.2642(2)$ & $0.0924(3)$ & $4.5(1)$ \\
\hline$C(6)$ & $1.2610(3)$ & $0.1920(2)$ & $0.1857(3)$ & $3.9(1)$ \\
\hline$C(7)$ & $1.2308(3)$ & $0.2033(2)$ & $0.3216(3)$ & $3.3(1)$ \\
\hline$C(8)$ & $1.2947(3)$ & $0.1456(2)$ & $0.4550(3)$ & $3.1(1)$ \\
\hline$C(9)$ & $1.3840(2)$ & $0.2126(2)$ & $0.6056(3)$ & $2.9(1)$ \\
\hline$C(10)$ & $1.2824(2)$ & $0.2885(2)$ & $0.6460(2)$ & $2.74(9)$ \\
\hline$C(11)$ & $0.8455(2)$ & $0.3211(2)$ & $0.4766(3)$ & $3.1(1)$ \\
\hline$C(12)$ & $0.7664(3)$ & $0.2797(2)$ & $0.3207(3)$ & $4.6(1)$ \\
\hline$C(13)$ & $0.6120(3)$ & $0.2932(2)$ & $0.2802(4)$ & $5.3(1)$ \\
\hline$C(14)$ & $0.5382(3)$ & $0.3457(2)$ & $0.3885(4)$ & $4.8(1)$ \\
\hline$C(15)$ & $0.6160(3)$ & $0.3864(2)$ & $0.5409(3)$ & $3.9(1)$ \\
\hline$C(16)$ & $0.7738(3)$ & $0.3732(2)$ & $0.5851(3)$ & $3.1(1)$ \\
\hline$C(17)$ & $1.1638(3)$ & $0.1111(2)$ & $0.6063(3)$ & $3.1(1)$ \\
\hline$C(18)$ & $1.0321(3)$ & $0.1112(2)$ & $0.6274(3)$ & $3.7(1)$ \\
\hline$C(19)$ & $1.0367(3)$ & $0.0614(2)$ & $0.7349(3)$ & $4.2(1)$ \\
\hline$C(20)$ & $1.1716(3)$ & $0.0087(2)$ & $0.8245(3)$ & $4.0(1)$ \\
\hline$C(21)$ & $1.3011(3)$ & $0.0064(2)$ & $0.8000(3)$ & $4.5(1)$ \\
\hline$C(22)$ & $1.2992(3)$ & $0.0566(2)$ & $0.6932(3)$ & $4.0(1)$ \\
\hline$C(23)$ & $1.1758(5)$ & $-0.0451(3)$ & $0.9419(5)$ & $5.7(2)$ \\
\hline$C(24)$ & $1.5505(3)$ & $0.1936(2)$ & $0.6784(3)$ & $3.2(1)$ \\
\hline$C(25)$ & $1.7619(3)$ & $0.2577(3)$ & $0.8777(5)$ & $4.6(1)$ \\
\hline$C(26)$ & $1.3015(2)$ & $0.3829(2)$ & $0.7618(3)$ & 3.1(1) \\
\hline$C(27)$ & $1.3426(6)$ & $0.4636(3)$ & $1.0304(5)$ & $6.1(2)$ \\
\hline
\end{tabular}

$B_{\mathrm{eq}}=(4 / 3) \sum_{i} \Sigma_{j} b_{i j} a_{i}^{*} a_{j}^{*}\left(\boldsymbol{a}_{i} \cdot \boldsymbol{a}_{j}\right)$.

Table 3 Selected bond lengths and angles (e.s.d. values in parentheses)

\begin{tabular}{cclc}
\hline Bond & Length $(\AA)$ & \multicolumn{1}{c}{ Bond } & Angle $\left(^{\circ}\right)$ \\
\hline$N(2)-N(3)$ & $1.369(2)$ & $\mathrm{C}(11)-\mathrm{N}(2)-\mathrm{N}(3)$ & $109.9(2)$ \\
$N(3)-N(4)$ & $1.299(3)$ & $\mathrm{N}(2)-\mathrm{N}(3)-\mathrm{N}(4)$ & $108.6(2)$ \\
$N(2)-\mathrm{C}(11)$ & $1.367(3)$ & & \\
$N(4)-C(16)$ & $1.374(3)$ & & \\
$\mathrm{C}(11)-\mathrm{C}(16)$ & $1.388(3)$ & & \\
\hline
\end{tabular}

Selected bond lengths and angles are recorded in Table 3. The chemical structure of $\mathbf{1}$ is cited in Fig. 1. The crystal structure of $\mathbf{1}$ is shown in Fig. 2, together with the atomic labeling scheme.

The crystal of $\mathbf{1}$ possesses the following four structural features: (a) The structure was identified as 11-aza-1-(1,2,3-1Hbenzotriazol-1-yl)-11-(4-methylphenyl)-tricyclo-
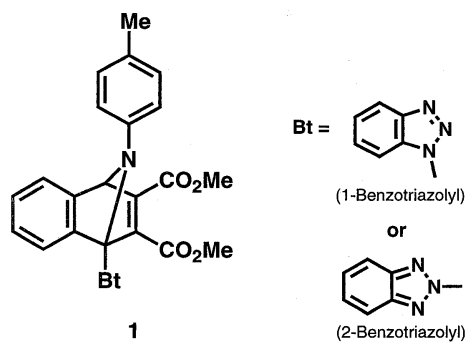

Fig. 1 Chemical structures of components.

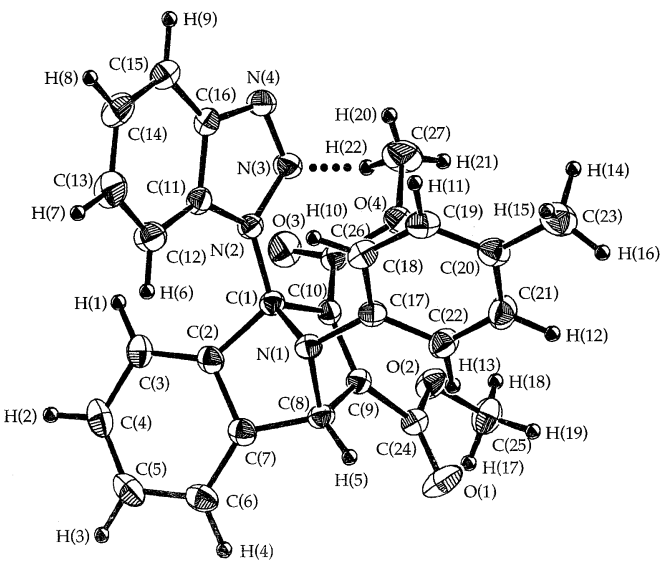

Fig. 2 The crystal structure of $\mathbf{1}$.

[5.2.1.0 $\left.0^{2,7}\right]$ undeca-2,4,6,9-tetraene-9,10-dioic acid dimethyl ester. (b) The observed bond angles and lengths in the structure are normal. (c) The benzene ring in the tricyclo framework and the benzotriazole plane were twisted with an angle of $115.83^{\circ}$, which would enable an interpretation of the unusual ${ }^{1} \mathrm{H}$ NMR chemical shift pattern in terms of diamagnetism. (d) Among intramolecular close contacts, those between benzotriazole and ester are characteristic $[\mathrm{N}(3) \cdots \mathrm{C}(26), 2.754(3) \AA ; \mathrm{N}(3) \cdots \mathrm{H}(22)$,

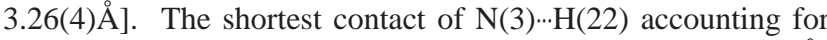
the rotation of the methyl group is estimated to be $3.10 \AA$ which might be reasonable as $\mathrm{C}-\mathrm{H} \cdot \cdots \mathrm{N}$-type hydrogen bonding.

\section{References}

1. I. Takahashi, M. Tsuzuki, T. Keumi, H. Kitajima, K. Isa, S. Hosoi, and Y. Tsuda, Chem. Pharm. Bull., 1994, 42, 947.

2. A. R. Katritzky, S. Rachwal, and G. J. Hitchings, Tetrahedron, 1991, 47, 2683

3. I. Takahashi, M. Tsuzuki, H. Yokota, T. Morita, H. Kitajima, and K. Isa, Heterocycles, 1996, 43, 71. 